\title{
Isolation and screening of cellulase producing bacterial strains from Cafeteria waste
}

\author{
Nitesh Kumar Saxena, Vikas Sharma ${ }^{1}$, Priyam Tyagi and Aarti Sharma \\ Molecular Genetics Lab, Centre for Cellular and Molecular Biology, Amity Institute of Biotechnology, Amity University, \\ Sector 125, Noida (U.P.) India \\ ${ }^{1}$ Department of Biotechnology, National Institute of Technology, Raipur (C.G.) India \\ Email : asharma11@amity.edu
}

Article Info : Received : 17.02.2018; Revised : 10.03.2018; Accepted : 24.03.2018

Cellulases are a group of enzymes that are involved in the breakdown of cellulose into simpler sugars. These enzymes have tremendous application in the production various products like pulp and paper, textiles etc. Plants, animals and various microorganisms like protozoans, bacteria and fungi naturally produce these enzymes. Since most industrial applications utilize fungi and bacteria for fermentation, this study was also designed to isolate cellulose producing bacterial strains. In this study, food waste was collected from cafeteria and used for isolation of cellulase producing bacterial strains. The waste was pulverized using mortar and pestle subsequently used for making serial dilution, followed by the spreading of diluents on nutrient agar plates. Morphologically distinct colonies were selected and streaked to obtain single colonies. Cellulase production potential of the selected 8 isolates was tested by streaking each strain on agar plates enriched with different concentration of carboxymethylcellulose (as the sole carbon source). The plates were incubated for 72-120 hours followed by staining with $1 \%$ (v/ v) Congo-red dye. Further the plates were decolourised by 1M Sodium chloride solution. A zone of clearance was observed around the bacterial strains capable of cellulase production because of their ability to hydrolyze cellulose. Further, the ability of the isolates to utilize varying concentration of CMC was tested in order to ascertain. The study resulted in isolation of 3 cellulase producing bacterial strains from cafeteria waste which can be utilized in cellulase production in the industries and also in agricultural waste management.

Key words : Cellulase, Cellulose hydrolysis, Waste, Paper, Pulp industry, Microbial product

How to cite this paper : Saxena, Nitesh Kumar, Sharma, Vikas, Tyagi, Priyam and Sharma, Aarti (2018). Isolation and screening of cellulase producing bacterial strains from Cafeteria waste. Asian J. Bio. Sci., 13 (1) : 28-31.DOI : 10.15740/HAS/AJBS/13.1/28-31. 